\title{
Increased radiation tolerance of CMOS sensors with small collection electrodes through accelerated charge collection
}

\author{
Valerio Dao* \\ CERN \\ E-mail: valerio.dao@cern.ch
}

\begin{abstract}
Mini-MALTA is a Monolithic Active Pixel Sensor prototype developed in the TowerJazz $180 \mathrm{~nm}$ CMOS imaging process, with a small collection electrode design $\left(3 \mu \mathrm{m}^{2}\right)$, and a small pixel size (36.4 $\mu \mathrm{m})$, on high resistivity substrates and large voltage bias. It targets the outermost layer of the ATLAS ITK Pixel detector for the HL-LHC. This design addresses the pixel inefficiencies observed in previous MALTA and TJ-Monopix prototypes. The implementation of a mask change in the fabrication or an additional implant allows to improve the charge collection performance at the edges of the pixel thus improving the radiation hardness properties. This contribution will present the results from characterisation in particle beam tests that show full efficiency up to $1 \cdot 10^{15} n_{e q} / \mathrm{cm}^{2}$.
\end{abstract}

Topical Workshop on Electronics for Particle Physics TWEPP2019

2-6 September 2019

Santiago de Compostela - Spain

\footnotetext{
* Speaker.
} 


\section{Introduction}

Several monolithic CMOS sensor technologies have been considered for the outer pixel layer of the ATLAS detector upgrade for the High-Luminosity LHC [1], requiring radiation tolerance to about $2 \cdot 10^{15} 1 \mathrm{MeV} n_{e q} / \mathrm{cm}^{2}$ and $70 \mathrm{Mrad}$. Monolithic CMOS sensors additionally allow to minimize scattering material for best tracking performance. The TowerJazz $180 \mathrm{~nm}$ CMOS imaging process with small, low capacitance collection electrodes offers a better combination of analog performance and power consumption. It allows full CMOS circuitry in the pixel, which in combination with a modification in the process allows full depletion of the epitaxial layer. First measurements performed on this technology showed improved radiation tolerance by an order of magnitude with respect to the un-modified process [2].

Following these results, the MALTA [3] large-scale prototype was designed and fabricated; it consists of a matrix of $512 \times 512$ squared pixels with $36.4 \mu \mathrm{m}$ pitch. The small electrode diameter of $3 \mu$ leads to an electrode capacitance of $5 \mathrm{fF}$ ensuring low noise operation and high $\mathrm{Q} / \mathrm{C}$ ratio in the analog input circuit ${ }^{1}$. The chip implements a novel asynchronous readout offering lower power consumption and higher matrix bandwidth due to no clock propagation in the pixel matrix and no latency from serial communication.

The prototype showed severe charge collection inefficiencies near the pixel edges after irradiation despite the process modification [3]. Detailed investigation including TCAD simulations [4] showed the low lateral electric field near the pixel borders significantly increases the collection time for signal charge generated in that area, especially for pixel pitches above $30 \mu \mathrm{m}$. Following these results a new prototype has been produced to address these issues: Mini-MALTA. This report contains the first experimental results using test beam on Mini-MALTA before and after the irradiation.

\section{Mini-MALTA}

Mini-MALTA [5] is reduced-size prototype (16x64 pixels) following the same solution as the MATLAS chip for what concerns pixel pitch and electrode size. Contrary to its predecessor, it implements an FIFO storing the end-of-column logic data and it transmits the information to the outside readout either via a 48 -bit $8 \mathrm{~d} / 10 \mathrm{~b}$ encoding at 1.2 Gbps or simple serial stream at 40 Mbps. Most notably, the Mini-MALTA sensor comprises eight different pixel flavours differing in analogue front-end design, reset mechanism and electrode/well geometries. They are implemented in the matrix as 8x16 pixel groups as illustrated in Figure 1 (left).

The sectors on the right side have an analog front end design identical to the one of MALTA while the sectors on the left implements an increase of $20 \%$ in the size of a key NMOC transistors in the analogue amplification circuit with the aim of reducing Random-Telegraph-Signal (RTS) noise. The bottom sectors ( $\mathrm{S} 0, \mathrm{~S} 4)$ contain the same type of sensor as MALTA. Sectors S1/S5 contains an additional production process compatible modification increasing the depth of the p-type implant at the corners of the chip (referred to as "extra deep p-well" in the following). Sectors S3/S7 implements a different process modification with reduced b-type implant in the corners (referred to

\footnotetext{
${ }^{1}$ In the case of a $25 \mu \mathrm{m}$ thick sensitive layer we expect a most probable ionization charge of around $1500 e^{-}$, leading to a change in voltage of around $50 \mathrm{mV}$
} 

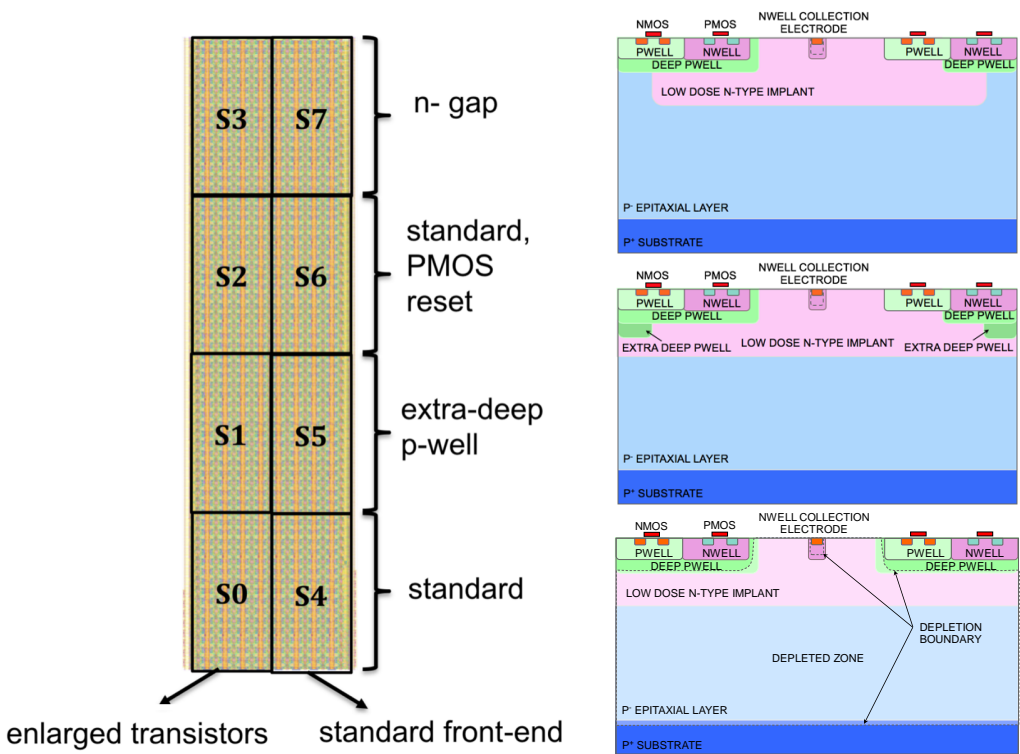

Figure 1: Layout of the Mini-MALTA pixel matrix at the read-out level and pixel sub-groups of $8 \times 16$ pixels (left). (right) Cross section of the TowerJazz process: the low dose n-implant removed (n-gap) at the edge of the pixel (top), extra deep p-well at the edge of the pixel (middle), and continuous n-layer (bottom).

as " $n$ - gap" in the following). The three types of process modifications are illustrated in Figure 1 $(\text { right })^{2}$. The latter modifications affect the electrical field at the edge of the pixel and from TCAD simulation and significantly improve the charge collection to the electrode after irradiation.

\section{Mini-MALTA threshold characterisation}

The threshold of the pixel matrix is controlled through dedicated on-chip 8-bit DACs which alter the performance of the pre-amplifier stage and in-pixel discriminator. The main threshold adjustment is carried out through the discriminator bias current ("IDB"), which is set globally for the full matrix. The threshold is measured for each pixel by injecting pulses with varying voltage amplitude on a in-pixel test capacitance $(0.23 \mathrm{fF})$ at the input of each preamplifier. The threshold is defined as the point at which $50 \%$ of the pulses is recorded and it is determined by fitting a Gauss error function to the hit occupancy S-curve; the sigma parameter of Gauss error function is used to extract the value of the equivalent noise charge (ENC). Figure 2 shows the measured threshold distributions for unirradiated and neutron irradiated Mini-MALTA samples, separately for sensor regions with standard (right plot) and enlarged transistors (left plot). The same default chip tuning configuration is used for all sensors which were operated at $-6 \mathrm{~V}$ substrate voltage and kept at $-20^{\circ} \mathrm{C}$. The gaussian fits are performed to the threshold distributions for each chip to better highlight its key characteristics. For regions with standard transistors, the average pixel threshold decreases from $570 e^{-}$(unirradiated) to $290 e^{-}\left(2 \cdot 10^{15} 1 \mathrm{MeV} n_{e q} / \mathrm{cm}^{2}\right)$ as a result of the change in the sensor capacitance. For the same level of irradiation, all the regions with enlarged transistors

\footnotetext{
${ }^{2}$ Sectors S2 and S6 are not considered in the current results.
} 

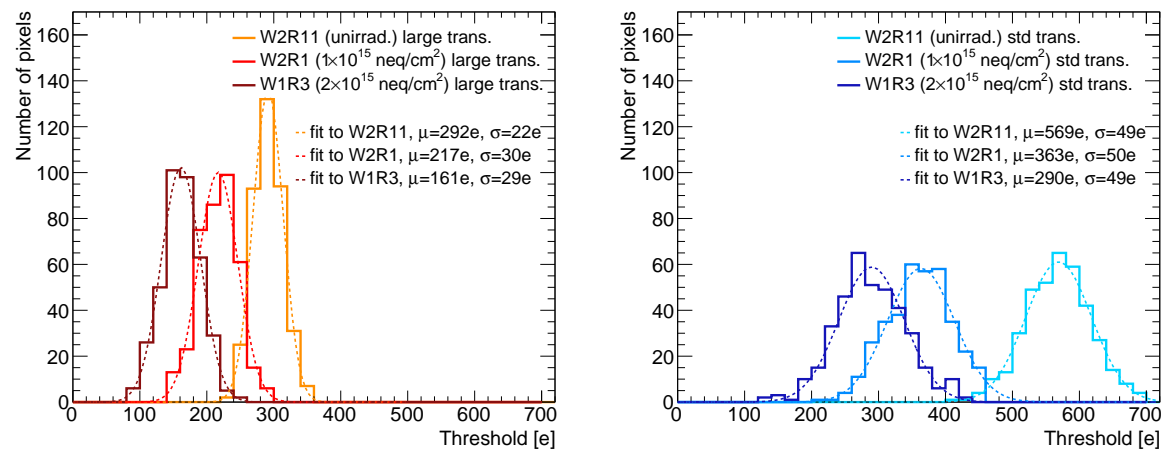

Figure 2: Pixel threshold distributions for unirradiated and neutron-irradiated Mini-MALTA samples at $1 \cdot 10^{15}$ and $2 \cdot 10^{15} 1 \mathrm{MeV} n_{e q} / \mathrm{cm}^{2}$. Gaussian fits to threshold distributions are shown as dashed lines. The same tuning configuration ( $\mathrm{IDB}=100$ ) was used in both unirradiated and irradiated sensors. Sensor regions with enlarged (left) and standard (right) transistors are shown.

shows value of threshold systematically lower than the regions with standard transistors by 300$150 e^{-}$; the threshold dispersion also halves for the regions with the enlarged transistor $\left(\sim 50 e^{-}\right.$to 20-30 $e^{-}$) further demonstrating the superior performance of this solution.

The mean value of the ENC distributions if fairly similar between regions with enlarged and standard transistors. Larger tails are observed in the regions with the standard transistors; this is attributed to Random Telegraph Signal noise (RTS) due to the use of minimal-size transistors in parts of the analog circuit. The average ENC value roughly double between unirradiated and irradiated sensors $\left(10 e^{-}\right.$to $\left.20 e^{-}\right)$.

\section{Test beam results}

The data presented in this study were recorded at the ELSA test beam facility at the University of Bonn using electron beams of $2.5 \mathrm{GeV}$. A two-arm beam telescope has been used containing each 3 planes hosting a MALTA chip. In between a cold box containing a device under test (DUT) together with one additional plane containing a MALTA chip positioned at $2 \mathrm{~cm}$ to the DUT downstream of the beam. The trigger signal was generated from the coincidence of signal in both arms of the telescope. Tracks are reconstructed by requiring a pixel 'hit' on the third plane of the telescope in front of the DUT, and hits in the first two planes after the DUT ${ }^{3}$. Adjacent pixel hits are combined into clusters. The fitted track direction is obtained taking into account multiple scattering by using the General Broken Lines (GBL) formalism [6]. The track trajectory calculation uses the material description of the DUT and all telescope planes as well as the electron beam energy for this estimation. The reconstructed track is then extrapolated to the plane of the DUT; after the planes alignment procedure, tracks are required to have $\chi^{2} / \mathrm{Ndof}^{4}$ smaller than 10 , the unbiased residual distribution between the track intercept point and the cluster position on the DUT reaches a width of $13.5 \mu \mathrm{m}$ in both $\mathrm{x}$ and $\mathrm{y}$ coordinates.

\footnotetext{
${ }^{3}$ The choice of using only a subset of the telescope planes in the reconstruction stems from optimisation studies related to the impact of the multiple scattering on the position resolution

${ }^{4}$ The number of degree of freedom of a track is $2 * \mathrm{~N}-4$ where $\mathrm{N}$ is the number of spacial points used in the fit
} 
The hit detection efficiency is defined as the fraction of clusters on the DUT matched to telescope tracks over the total number of tracks; a cluster in DUT is matched to a track if the distance between the position of a track interpolated to the plane of the DUT and the position of the centre of the cluster is smaller than $100 \mu \mathrm{m}$. The overall efficiency is quoted for each sector after excluding an area at his border of the size of one pixel; this takes into account fiducial area effects due to the finite resolution of the tracks. For the same reason, tracks with hit predictions around pixels non functioning or disabled because of large noise rate (radius of $36.4 \mu \mathrm{m}$ around the pixel centre) are rejected from the efficiency analysis.

Figure 3 (left) shows the efficiency as a function of the track position in the DUT plane for an 1 . $10^{15} 1 \mathrm{MeV} n_{e q} / \mathrm{cm}^{2}$ neutron irradiated Mini-MALTA sensor ("W2R1") operated at a configuration producing $200 e^{-}$on the enlarged transistors section and $340 e^{-}$on the standard transistors sector. The DUT was kept at a temperature of $-20^{\circ} \mathrm{C}$. Table 1 lists the measured efficiency at given threshold values for all Mini-MALTA sensors used in the beam tests in dependency of the implant configuration and front-end amplifier design. While unirradiated sensor shows efficiency larger that $97 \%$ for all the sectors, after the irradiation to $1 \cdot 10^{15} \mathrm{MeV} \mathrm{n} \mathrm{n}_{e q} / \mathrm{cm}^{2}$ a more diverse pattern emerges. The average efficiency significantly decreases for regions with standard transistors due to the lower gain and higher effective threshold in these sectors: it reaches $78.8 \%$ in the region with no extra sensor modification and $\sim 87 \%$ in sectors with extra deep p-well and n- gap. While the modifications to the implant proof effective, the overall efficiency is still significantly affected by the high threshold. The regions with enlarged transistors allows to reach a lower threshold for the sam chip configuration resulting an an overall efficiency above $97 \%$ the two modified processes, confirming the results of the simulation predictions.

\begin{tabular}{|c|c|c|c|c|c|}
\hline Chip ID & $\mathrm{EPI}[\mu \mathrm{m}]$ & $\begin{array}{c}\text { Fluence } \\
{\left[1 \mathrm{MeV} \mathrm{n}_{e q} / \mathrm{cm}^{2}\right]} \\
\end{array}$ & $\begin{array}{c}\text { Process } \\
\text { modification } \\
\end{array}$ & $\begin{array}{c}\text { Eff. enlarged trans. [\%] } \\
\text { / threshold }\left[\mathrm{e}^{-}\right]\end{array}$ & $\begin{array}{l}\text { Eff. (std trans.) }[\%] \\
\text { / threshold }\left[\mathrm{e}^{-}\right]\end{array}$ \\
\hline W2R11 & 30 & unirrad. & $\begin{array}{c}\mathrm{n}^{-} \text {gap } \\
\text { extra deep p-well } \\
\text { continuous } \mathrm{n}^{-}\end{array}$ & $\begin{array}{l}99.6 \pm 0.1 / 200 \mathrm{e}^{-} \\
99.6 \pm 0.1 / 200 \mathrm{e}^{-} \\
99.6 \pm 0.1 / 200 \mathrm{e}^{-}\end{array}$ & $\begin{array}{l}99.1 \pm 0.1 / 380 \mathrm{e}^{-} \\
98.9 \pm 0.1 / 380 \mathrm{e}^{-} \\
97.9 \pm 0.1 / 380 \mathrm{e}^{-}\end{array}$ \\
\hline W2R1 & 30 & $1 \times 10^{15}$ & $\begin{array}{c}\mathrm{n}^{-} \text {gap } \\
\text { extra deep p-well } \\
\text { continuous } \mathrm{n}^{-}\end{array}$ & $\begin{array}{l}97.6 \pm 0.1 / 105 \mathrm{e}^{-} \\
97.9 \pm 0.1 / 105 \mathrm{e}^{-} \\
91.9 \pm 0.1 / 105 \mathrm{e}^{-}\end{array}$ & $\begin{array}{l}86.5 \pm 0.1 / 210 \mathrm{e}^{-} \\
87.0 \pm 0.1 / 210 \mathrm{e}^{-} \\
78.8 \pm 0.2 / 210 \mathrm{e}^{-}\end{array}$ \\
\hline W4R2 & 25 & $1 \times 10^{15}$ & $\begin{array}{c}\mathrm{n}^{-} \text {gap } \\
\text { extra deep p-well } \\
\text { continuous } \mathrm{n}^{-} \\
\end{array}$ & $\begin{array}{l}98.8 \pm 0.1 / 120 \mathrm{e}^{-} \\
99.2 \pm 0.1 / 120 \mathrm{e}^{-} \\
95.8 \pm 0.1 / 120 \mathrm{e}^{-}\end{array}$ & $\begin{array}{l}90.7 \pm 0.1 / 275 \mathrm{e}^{-} \\
92.5 \pm 0.1 / 275 \mathrm{e}^{-} \\
79.4 \pm 0.2 / 275 \mathrm{e}^{-}\end{array}$ \\
\hline
\end{tabular}

Table 1: Summary of the efficiency measurements in each sector for various Mini-MALTA chips. All chips were operated at low threshold settings. The thickness of the depletion region (epitaxial layer) for each chip is also reported. The uncertainties listed are statistical.

\section{Conclusions}

Sensor production process modifications and changes in the size of the analogue front-end 

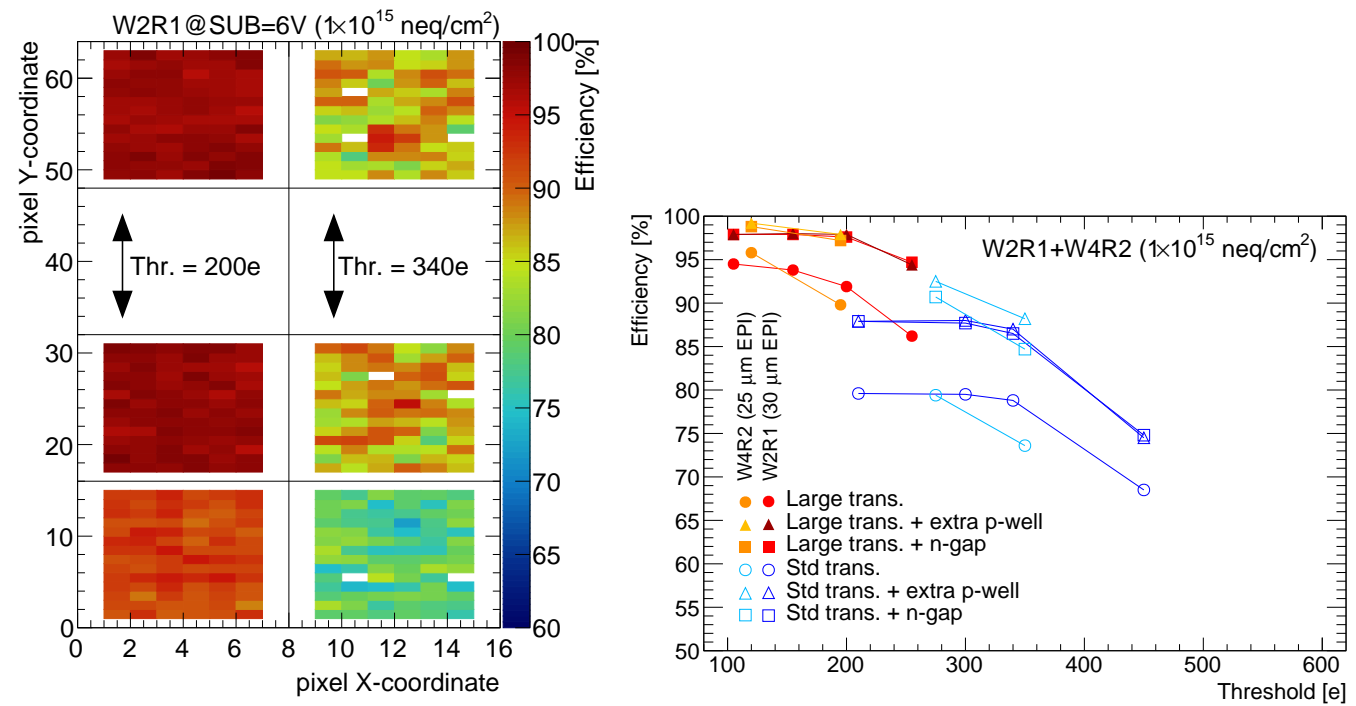

Figure 3: (left) 2D efficiency map for an irradiated Mini-MALTA samples at $1 \cdot 10^{15} 1 \mathrm{MeV}$ $n_{e q} / \mathrm{cm}^{2}$. Different sensor regions are visible: standard MALTA-like (bottom part of each chip), modified with extra deep p-well (middle part) and modified with extra n-gap (top part). Results are also shown for sensor regions with standard (right side of each chip) and enlarged (left side) transistors. The chips were operated at $-6 \mathrm{~V}$ substrate voltage and $-20^{\circ} \mathrm{C}$, and were tuned for low threshold. (right) Efficiency versus threshold mean for neutron irradiated Mini-MALTA samples at $1 \cdot 10^{15} 1 \mathrm{MeV} n_{e q} / \mathrm{cm}^{2}$. Different sensor regions are presented: standard MALTA-like (circles), modified with extra deep p-well (triangles) and modified with extra n-gap (rectangles). Results are also shown for sensor regions with standard (open markers) and enlarged (full markers) transistors, as well as for sensors with different epitaxial layer thicknesses: $25 \mu \mathrm{m}$ (orange or light blue symbols) and $30 \mu \mathrm{m}$ (red or dark blue symbols).

transistors have lead to Mini-MALTA CMOS prototype to be full efficient after radiation dose of $1 \cdot 10^{15} 1 \mathrm{MeV} n_{e q} / \mathrm{cm}^{2}$.

\section{References}

[1] ATLAS collaboration, Technical Design Report for the ATLAS Inner Tracker Pixel Detector, Tech.Rep. CERN-LHCC-2017-021. ATLAS-TDR-030, 2017.

[2] W. Snoeys et al., A process modification for CMOS monolithic active pixel sensors for enhanced depletion, timing performance and radiation tolerance, Nucl. Instrum. Meth. A871 (2017) $90 \nsupseteq 96$.

[3] R. Cardella et al., MALTA: an asynchronous readout CMOS monolithic pixel detector for theATLAS High-Luminosity upgrade, JINST 14 (2019) C06019.

[4] M. Munker et al., Simulations of CMOS pixel sensors with a small collection electrode, improved for a faster charge collection and increased radiation tolerance, JINST 14 (2019) C05013, [1903.10190].

[5] M. Dyndal et al., Mini-MALTA: Radiation hard pixel designs for small-electrode monolithic CMOS sensors for the High Luminosity LHC, arXiv:1909.11987

[6] M. Kiehn et al., Proteus beam telescope reconstruction, 2019. 10.5281/zenodo.2586736. 